\title{
Effects of High Hydrostatic Pressure Extract of Korean Fresh Ginseng on Hepatic Lipid Accumulation and AMPK Activation in HepG2 Cells
}

\author{
Mak-Soon Lee ${ }^{1, \#}$, Yoonjin Shin ${ }^{1, \#}$, Sunyoon Jung ${ }^{1}$, Chong-Tai Kim², In-Hwan Kim³, Yangha Kim, ${ }^{1, *}$ \\ ${ }^{1}$ Department of Nutritional Science and Food Management, Ewha Womans University, Seoul, Republic of Korea \\ ${ }^{2}$ Research Group of Convergence Technology, Korea Food Research Institute, Seongnam, Gyeonggi, Republic of Korea \\ ${ }^{3}$ Department of Food and Nutrition, Korea University, Seoul, Republic of Korea \\ \# These authors contributed equally to this work and should be considered co-first authors. \\ *Corresponding author: yhmoon@ewha.ac.kr
}

Received January 10, 2015; Revised January 19, 2015; Accepted January 22, 2015

\begin{abstract}
Ginseng is widely used as a medicinal herb and has demonstrated effects against liver diseases. The aim of this study is to investigate the hypolipidemic effects of the high hydrostatic pressure extract of Korean fresh ginseng (HEG) on hepatic lipid accumulation in HepG2 cells. The intracellular triglyceride and cholesterol contents were determined using enzymatic colorimetric methods. The mRNA levels of fatty acid synthase (FAS) and 3hydroxy-3-methyl-glutaryl CoA reductase (HMGCR) were assayed by quantitative real-time PCR. The activity of AMP-activated protein kinase (AMPK) was measured with an AMPK kinase assay kit. HEG significantly reduced hepatic triglyceride and cholesterol contents in HepG2 cells. Furthermore, HEG suppressed the expression of FAS, a key enzyme in fatty acid synthesis, and HMGCR, a rate-limiting enzyme in hepatic cholesterol synthesis. Additionally, HEG increased the activity of AMPK, a major regulator of lipid metabolism. These results suggest that HEG reduces hepatic lipid accumulation with inhibition of FAS and HMGCR expression and stimulation of AMPK activity in HepG2 cells. Consequently, HEG may be beneficial as a functional food ingredient to improve various hepatic diseases by reducing hepatic lipid accumulation.
\end{abstract}

Keywords: korean fresh ginseng, high hydrostastic pressure extract, lipid accumulation, FAS, HMGCR, AMPK

Cite This Article: Mak-Soon Lee, Yoonjin Shin, Sunyoon Jung, Chong-Tai Kim, In-Hwan Kim, and Yangha Kim, "Effects of High Hydrostatic Pressure Extract of Korean Fresh Ginseng on Hepatic Lipid Accumulation and AMPK Activation in HepG2 Cells.” Journal of Food and Nutrition Research, vol. 3, no. 1 (2015): 40-45. doi: 10.12691/jfnr-3-1-7.

\section{Introduction}

The liver plays an important role in the regulation of whole-body energy metabolism as a major organ for fatty acid and cholesterol synthesis. Hepatic lipid accumulation is associated with obesity, insulin resistance, hyperlipidemia, and metabolic disorders [1,2]. Approximately $20-30 \%$ of adults in western countries are estimated to have nonalcoholic hepatic disease [3]. Prevention of these diseases using bioactive functional ingredients from natural foods is likely to be the most effective strategy for overcoming disease as promotion of good health.

AMP-activated protein kinase (AMPK) is a metabolic regulator that modulates energy balance by raising the intracellular AMP/ATP ratio. Activation of AMPK in the liver inhibits lipid biosynthetic pathways by repressing fatty acid and sterol synthesis. AMPK inactivates key lipid biosynthesis enzymes, such as fatty acid synthase (FAS) and 3-hydroxy-3-methyl-glutaryl CoA reductase (HMGCR) [4]. Therefore, AMPK might be an attractive therapeutic target for intervention in metabolic diseases including hyperlipidemia.

Ginseng is used extensively as a traditional herbal medicine in Asian countries due to its curative effects against various human diseases such as inflammation, cancer, obesity, diabetes, hypertension, and arteriosclerosis $[5,6,7]$. The beneficial effects of ginseng have attributed to ginsenosides, the primary bioactive ingredients in ginseng [8]. Approximately 40 different types of ginsenosides have been identified in Korean ginseng [9]. The amount of major ginsenosides found in high hydrostatic pressure extracts of Korean ginseng is a $45 \%$ higher than in extracts using conventional methods [10]. It has been shown that ginseng extract prevents hyperlipidemia through AMPK activation and through the inhibition of acetyl-CoA carboxylase- $\alpha$ (ACC- $\alpha$ ) and sterol-regulatory element binding protein 1 (SREBP-1) [11,12]. However, the effects of high hydrostatic pressure extract of ginseng (HEG) on hepatic lipid metabolism and AMPK activation in HepG2 cells have yet to be examined.

Therefore, the aim of this study was to identify the hypolipidemic effects of HEG in HepG2 cells. We hypothesized that treatment with HEG may increase 
AMPK activity, which inhibits the expression of FAS and HMGCR leading to a decrease in hepatic triglyceride and cholesterol accumulation.

\section{Materials and Methods}

\subsection{Materials}

The human HepG2 cell line was obtained from American Type Culture Collection (Manassas, VA, USA). Dulbecco's modified Eagle's medium (DMEM), phosphate-buffered saline, pH 7.4 (PBS), fetal bovine serum (FBS), and penicillin-streptomycin, TRIzol reagent, and M-MLV reverse transcriptase were purchased from Gibco/Invitrogen (Grand Island, NY, USA). The Universal SYBR Green PCR Master Mix was obtained from Qiagen (Valencia, CA, USA). A cell count kit-8 (CCK-8) was purchased from Dojindo Laboratories (Kumamoto, Japan). Assay kits for cholesterol and triglyceride were obtained from Asan Pharmaceutical (Seoul, Korea). The AMPK Kinase Assay kit was purchased from Cyclex (Nagano, Japan). A bicinchoninic acid (BCA) protein assay kit was obtained from Thermo Scientific (Pittsburgh, PA, USA). Hoechst 33342, triton X-100 and compound c were purchased from Sigma-Aldrich (Louis, MO, USA).

\subsection{Preparation of HEG}

HEG was kindly supplied by the Korea Food Research Institute (Songnam, Gyeonggi, Korea) [13]. Six-year-old Korean fresh ginseng (Panax ginseng) roots from GimpoPaju Ginseng Agricultural Cooperative (Gimpo, Gyeonggi, Korea) were used for the HEG preparation. To prepare the extract, ginseng root suspension was poured into plastic bags with $25 \mathrm{~mL}$ of each enzyme (Termamyl $120 \mathrm{~L}$, Celluclast $1.5 \mathrm{~L}$ and Viscozyme L) and transferred to a programmable high-pressure treatment apparatus (TFS-10 L, Innoway Co., Bucheon, Korea), maintained at $100 \mathrm{MPa}$ pressure for $24 \mathrm{~h}$ at $50^{\circ} \mathrm{C}$. After incubation, the extract was heated at $100^{\circ} \mathrm{C}$ for $10 \mathrm{~min}$ to inactivate the enzyme. When extraction was complete, the cooled extract was centrifuged at $11,000 \times \mathrm{g}$ for $10 \mathrm{~min}$, and the supernatant was filtered using Whatman No. 4 filter paper. The filtrate was freeze-dried and used as HEG. The contents of major ginsenosides in HEG were as follows: Rg1, 10.7\%; Re, 10.5\%; Rf, 4.3\%; Rh1+Rg2, 1.6\%; Rb1, 31.2\%; Rc, 17.5\%; Rb2, 13.1\%; Rd, 4.6\%; F2, 1.0\%; Rg3, 1.6\%; Compound K, 0.8\%; Rg5, 1.2\%; Rk1, 0.8\%; Rh2, 0.3\% [13].

\subsection{Cell Culture}

Human HepG2 cells were cultured in DMEM supplemented with $10 \%(\mathrm{v} / \mathrm{v})$ FBS and penicillinstreptomycin $(100 \mathrm{U} / \mathrm{mL})$ at $37^{\circ} \mathrm{C}$ and $5 \% \mathrm{CO}_{2}$. Cells were treated with different concentrations of HEG in serum-free media for 6 and $24 \mathrm{~h}$. Control cells were treated without HEG. All measurements were performed in triplicate for each treatment.

\subsection{Cytotoxicity Assay}

Cell viability was determined by the WST-8 [2-(2-methoxy4-nitropheyl)-3-(4-nitrophenyl)-5-(2,4-dinitrophenyl)-2Htetrazolium, monosodium salt] assay, using a CCK-8 kit according to the manufacturer's instructions. The assay is based on the cleavage of the WST-8 tetrazolium salt to formazan by cellular mitochondrial dehydrogenase. Cell viability was determined following culture in 96-well plates at a seeding density of $10^{4}$ cells/well. Cells were treated with 0 (control), 1, 10, 50,100, or $500 \mu \mathrm{g} / \mathrm{mL}$ of HEG, and incubated for 6,24 or 48 h at $37^{\circ} \mathrm{C}$. WST-8/1methoxy-phenazine methosulfate solution was added to each well and incubated for $3 \mathrm{~h}$ at $37^{\circ} \mathrm{C}$. Absorbance at $450 \mathrm{~nm}$ was measured using a Varioskan plate reader (Thermo Electron, Waltham, MA, USA). Values are expressed as a percentage of the control cells without HEG supplementation.

\subsection{Hoechst 33342 Staining}

After treatment with 0 (control) and $50 \mu \mathrm{g} / \mathrm{mL}$ of HEG for $24 \mathrm{~h}$, the cells were washed with PBS and fixed with $10 \%$ formalin for $4 \mathrm{~h}$ at room temperature. Fixed cells were washed with PBS and stained with $10 \mu \mathrm{M}$ of Hoechst 33342 stain for $30 \mathrm{~min}$. Following staining with Hoechst 33342, cells were observed using a fluorescence microscope (Olympus Corporation, Tokyo, Japan) at 100× magnification.

\subsection{Triglyceride and Cholesterol Assay}

Triglyceride and cholesterol assay was performed as described previously [14] using Triglyceride and Cholesterol Assay kit in accordance with the manufacturer's instructions. Cells were lysed in a lysis buffer consisting of $1 \%$ Triton $\mathrm{X}-100$ in PBS, and cellular triglyceride and cholesterol were measured using enzymatic colorimetric assay kits. The cellular protein concentration was determined using a BCA protein assay kit. Cellular cholesterol and triglyceride were normalized to cellular protein content.

\subsection{Quantitative Real-time Polymerase Chain Reaction (PCR)}

Total RNA was extracted from HepG2 cells using TRIzol reagent. Complimentary DNAs were synthesized from $4 \mu \mathrm{g}$ of RNA using M-MLV reverse transcriptase. After cDNA synthesis, quantitative real-time PCR was performed in $20 \mu \mathrm{L}$ of Universal SYBR Green PCR Master Mix using a fluorometric thermal cycler (Corbett Research, Mortlake, NSW, Australia). Primers were designed using an on-line program (primer3_http://www.cgivo.2) [15]. The sequences of the sense and antisense primers used for amplification were as follows: FAS, 5'-GACGTCTGCAAGCCCAAGTA-3' and 5'-CATCGTCTCCACCAAAATGC-3'; HMGCR, 5'ACTTATGGCAGCATTGGCAG-3' and 5'ACTGTCGGGCTATTCAGGCT-3'; $\beta$-actin, $5^{\prime}-$ GGACCTGACTGACTACCTCA-3' and 5'GCACAGCTTCTCCTTAATGT-3'. The ${ }^{\Delta \Delta} \mathrm{Ct}$ method was used for relative quantification [16]. The ${ }^{{ }^{\Delta}} \mathrm{Ct}$ value for each sample was determined by calculating the difference between the $\mathrm{Ct}$ value of the target gene and the $\mathrm{Ct}$ value of the $\beta$-actin reference gene. The normalized level of expression of the target gene in each sample was calculated using the formula $2^{-\Delta \Delta \mathrm{Ct}}$. Values were expressed as a fold of the control.

\subsection{AMPK Activity Assay}

AMPK activity assay was performed as described previously [17], using an AMPK Kinase Assay kit in 
accordance with the manufacturer's instructions. Briefly, samples were incubated for $30 \mathrm{~min}$ at $30^{\circ} \mathrm{C}$ in a precoated plate using a substrate peptide that corresponded to mouse insulin receptor substrate-1 (IRS-1). AMPK activity was measured by monitoring the phosphorylation of Ser 789 on IRS-1 using an anti-mouse phospho-Ser 789 IRS-1 monoclonal antibody and peroxidase-coupled anti-mouse IgG. Conversion of the chromogenic substrate tetramethylbenzidine was quantified by measuring absorbance at $450 \mathrm{~nm}$. Protein was determined using a BCA protein assay kit. Values for AMPK activity were expressed as a fold increase over the control.

\subsection{Statistical Analysis}

Values are expressed as means \pm standard error of the mean (SEM). Statistical analyses were performed using SPSS Statistic software version 19.0 (IBM corporation, Armonk, NY, USA). Significant differences among treatment groups were analyzed using an unpaired Student's twotailed t test and a one-way analysis of variance followed by post hoc Tukey's multiple comparison tests. $P<0.05$ was taken to indicate a significant difference.

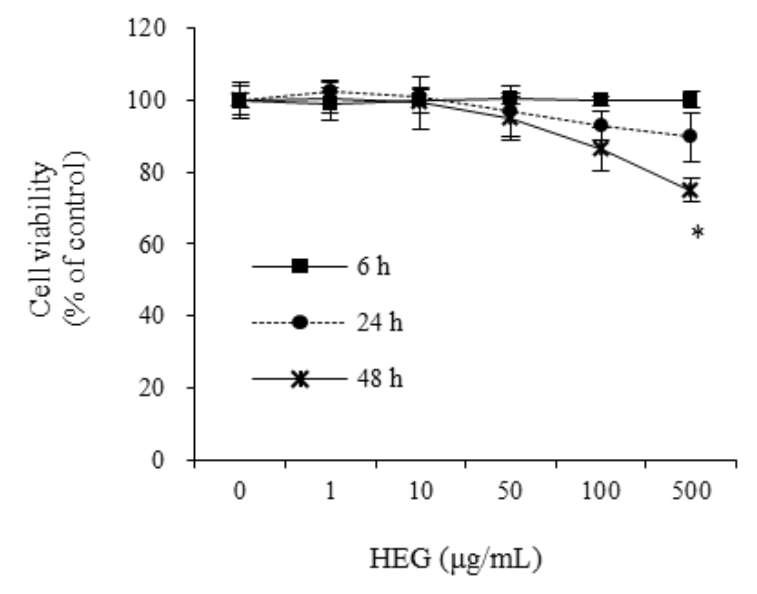

Figure 1. Effect of HEG on cell viability in HepG2 cells. Data are presented as mean \pm SEM $(n=3)$ of experiments performed in triplicate. ${ }^{*} P<0.05$. HEG, high hydrostatic pressure extract of ginseng

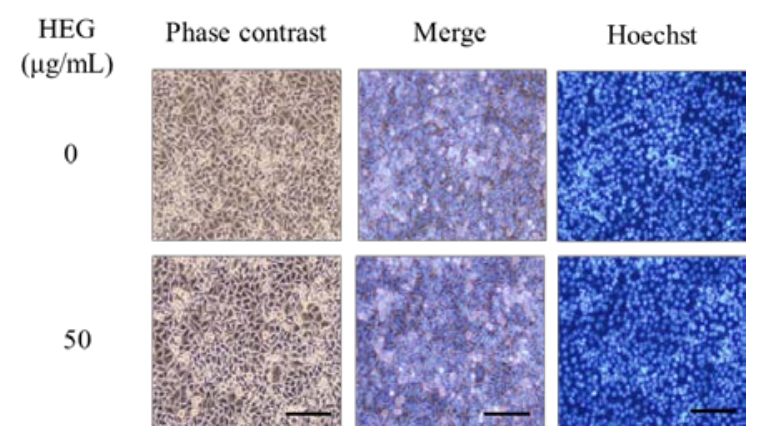

Figure 2. (Color online) Effect of HEG on apoptosis in HepG2 cells. Scale bar $=100 \mu \mathrm{m}$. HEG, high hydrostatic pressure extract of ginseng

\section{Results}

\subsection{Effect of HEG on HepG2 Cell Viability}

To investigate the potential cytotoxic effects of HEG, HepG2 cells were treated in various concentrations (1-500 $\mu \mathrm{g} / \mathrm{mL}$ ) of HEG for 6,24 , and $48 \mathrm{~h}$ at $37^{\circ} \mathrm{C}$. Cytotoxicity was unaffected by $50 \mu \mathrm{g} / \mathrm{mL}$ of HEG after $48 \mathrm{~h}$ incubation (Figure 1). However, high doses (100-500 $\mu \mathrm{g} / \mathrm{mL})$ of HEG decreased viability by $13.7-25.0 \%$ in $48 \mathrm{~h}$, respectively, compared to the untreated control. The effects of HEG on apoptosis induction in HepG2 cells were examined by the Hoechst 33258 dye-staining method. Cells were treated with 0 (control) or $50 \mu \mathrm{g} / \mathrm{mL}$ of HEG for $24 \mathrm{~h}$. The number of apoptotic cells treated with $50 \mu \mathrm{g} / \mathrm{mL}$ of HEG was similar to those in untreated control (Figure 2). Thus, HEG was determined to be nontoxic to the cells at concentrations below $50 \mu \mathrm{g} / \mathrm{mL}$ and period of treatment evaluated.

\subsection{Effect of HEG on Lipid Accumulation}

The effect of HEG on lipid accumulation was measured by the quantification of intracellular triglyceride and cholesterol contents in HepG2 cells. Cells were treated with 0 (control), 10, or $50 \mu \mathrm{g} / \mathrm{mL}$ of HEG for 6 and $24 \mathrm{~h}$. The amounts of intracellular triglyceride and cholesterol in the presence of $50 \mu \mathrm{g} / \mathrm{mL}$ of HEG were significantly decreased by $15.9 \%$ and $16.6 \%$, respectively, in comparison with the control after $24 \mathrm{~h}$ incubation but did not affect at $6 \mathrm{~h}$ (Figure $3 \mathrm{~A}$ and Figure 3B).
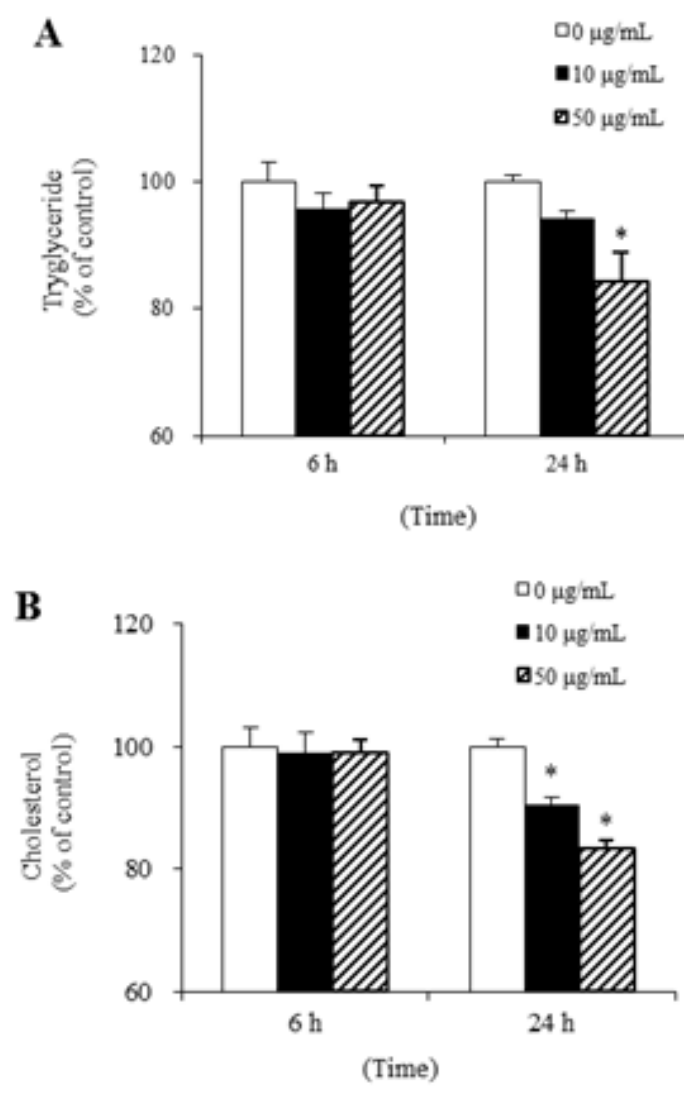

Figure 3. Effect of HEG on intracellular triglyceride (A) and cholesterol (B) content in HepG2 cells. Data are presented as mean \pm SEM $(n=3)$ of experiments performed in triplicate. ${ }^{*} P<0.05$. HEG, high hydrostatic pressure extract of ginseng

\subsection{Effects of HEG on FAS and HMGCR Expression}

The mRNA levels of FAS and HMGCR were investigated to understand the underlying mechanism behind the hypolipidemic effect of HEG in HepG2 cells. 
Cells were treated with 0 (control), 1,10 , or $50 \mu \mathrm{g} / \mathrm{mL}$ of HEG for $24 \mathrm{~h}$. The mRNA expression of FAS, a key enzyme of fatty acid biosynthesis, showed significant reductions of 43 or $86 \%$ with treatments of 10 or 50 $\mu \mathrm{g} / \mathrm{mL}$ of $\mathrm{HEG}$, respectively, as compared to control (Figure 4A). The mRNA expression of HMGCR, a rate limiting enzyme in cholesterol synthesis, was similarly decreased by 49 or $74 \%$ with treatments of 10 or 50 $\mu \mathrm{g} / \mathrm{mL}$ of HEG, respectively, as compared to control (Figure 4B).
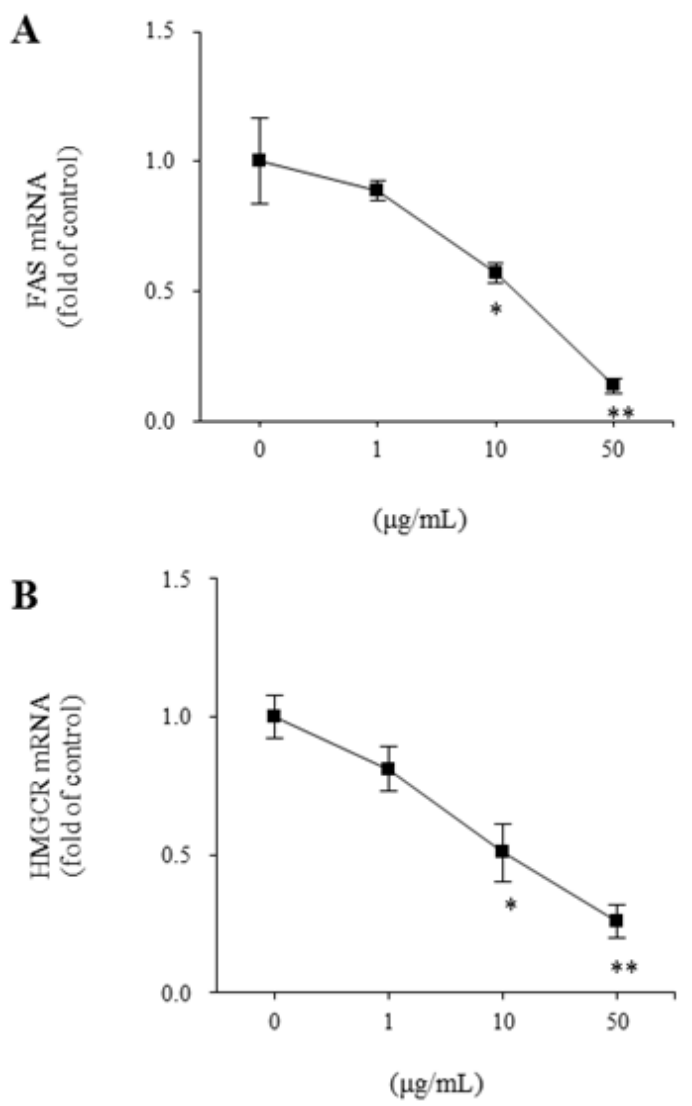

Figure 4. Effect of HEG on FAS (A) and HMGCR (B) expression in HepG2 cells. Data are presented as mean $\pm \operatorname{SEM}(n=3)$ of experiments performed in triplicate. ${ }^{*} P<0.05,{ }^{* *} P<0.01$. HEG, high hydrostatic pressure extract of ginseng; FAS, fatty acid synthase; HMGCR, 3hydroxy-3-methyl-glutaryl CoA reductase

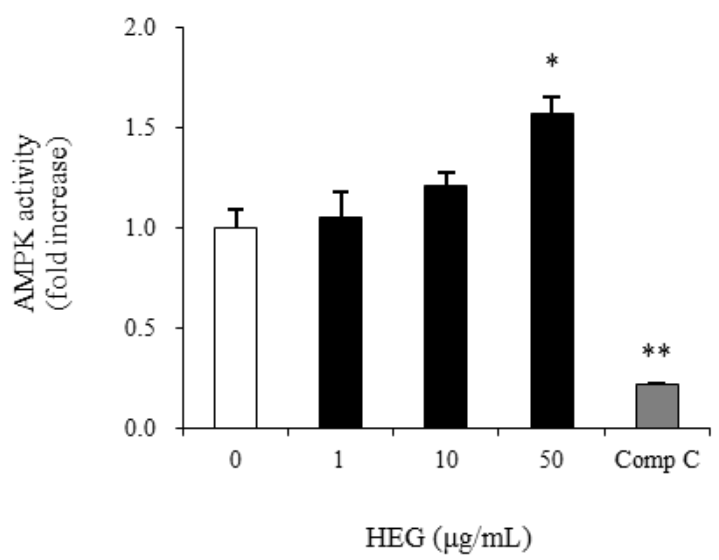

Figure 5. Effect of HEG on AMPK activity in HepG2 cells. Data are presented as mean $\pm \operatorname{SEM}(n=3)$ of experiments performed in triplicate. ${ }^{*} P<0.05,{ }^{* *} P<0.01$. HEG, high hydrostatic pressure extract of ginseng; AMPK, AMP-activated protein kinase

\subsection{Effect of HEG on AMPK Activation}

To evaluate whether HEG affects the activity of AMPK, which modulates lipid metabolism by inhibiting FAS and HMGCR expression, cells were treated with 0 (control), 1 , 10 , or $50 \mu \mathrm{g} / \mathrm{mL}$ of HEG for $24 \mathrm{~h}$. The AMPK activity in the presence of $50 \mu \mathrm{g} / \mathrm{mL}$ of HEG significantly was increased 1.57-fold compared with contro in HepG2 cells (Figure 5).

\section{Discussion}

Lipid accumulations within the liver have been proposed to cause obesity, diabetes, and fatty liver disease [18]. Ginseng is a widely used traditional medicine with demonstrated effects against various human diseases. The major bioactive ingredients of ginseng are ginsenosides, a diverse group of steroidal saponins with beneficial effects on health [19]. The aim of this study was to investigate the effects of HEG on hepatic lipid accumulation, particularly whether HEG affects FAS and HMGCR expression through the activation of AMPK.

To investigate the effects of HEG on hepatic lipid accumulation, the intracellular triglyceride and cholesterol contents in HepG2 cells were measured. Our results indicate that HEG significantly reduces triglyceride and cholesterol levels in HepG2 cells. A previous study indicated that treatment with fermented ginseng reduced triglyceride level with AMPK activation in HepG2 cells [20]. In addition, Song et al. reported that Korean Red ginseng extract decreased in the serum cholesterol level of mice fed a high-fat diet [21]. These results suggest that HEG may be beneficial in reducing hepatic lipid accumulation.

The mRNA expression of lipogenic genes, such as FAS and HMGCR were evaluated to understand the mechanism of the hypolipidemic effect of HEG in HepG2 cells. In this study, we found that HEG down-regulated both FAS and HMGCR expression in a dose-dependent manner. FAS is a key enzyme in the de novo lipogenesis pathway. It catalyzes all the steps in the conversion of malonyl-CoA to palmitate [22]. HMGCR plays a critical role in the transcriptional regulation of genes involved in the lipogenic pathway. It is responsible for catalyzing the principal regulatory step in the biosynthetic pathway of cholesterol [23]. In essence, the major sites of regulation within the pathways of fatty acid and cholesterol syntheses are catalyzed by FAS and HMGCR. A previous study reported that Korean white ginseng extract decreased FAS expression in white adipose tissue of high fat diet-induced obese mice [24]. In another study, supplementation of Korean ginseng powder was decreased mRNA expression of HMGCR in avian liver [25]. Similar to these results, our study showed that HEG down-regulated both FAS and HMGCR expression in HepG2 cells. Therefore, it can be postulated that hypolipidemic effect of HEG was partially associated with the inhibition of FAS and HMGCR expression.

AMPK is an enzyme that regulates energy homeostasis. Activated AMPK inhibits lipid biosynthetic pathways through down-regulation of lipogenic genes such as FAS and HMGCR [26]. There are several reports that ginsenosides improve lipid profiles though AMPK 
activation [14,27,28]. Ginsenoside Re attenuated lipid levels via AMPK activation in HepG2 cells and in high-fat diet fed mice [27]. The ginsenoside Rg3 reduced hepatic lipid accumulation by decreasing the expression of HMGCoA reductase and stimulating the activation of AMPK in HepG2 cells [14]. Compound $K$, a metabolite of ginsenoside, decreased hepatic triglyceride levels by regulating the expression of genes involved in lipogenesis and lipolysis via AMPK activation [28]. Consistent with these results, our study showed that HEG increases AMPK activation in HepG2 cells. Thus, it may be speculated that AMPK activation was partially involved in the inhibitory effect of HEG on hepatic lipid accumulation.

\section{Conclusion}

In conclusion, our results suggest that HEG may have beneficial effects on hepatic lipid accumulation in HepG2 cells. It is likely that the hypolipidemic effect of HEG may be related to activation of AMPK and to the inhibition of FAS and HMGCR expression. Thus, HEG may be useful as a potent lipid-lowering agent for improving various hepatic diseases.

\section{Acknowledgement}

This study was supported by the National Research Foundation of Korea (NRF) funded by the Korean Government (MOE) (No. 2013R1A1A2009522) and the Science Listening Post Project(SLP-04-003) through National Research Council of Science \& Technology (NST) of Korea.

\section{Competing Interests}

The authors have no competing interests.

\section{Abbreviations}

HEG: high hydrostatic pressure extract of ginseng; FAS: fatty acid synthase; HMGCR: 3-hydroxy-3-methyl-glutaryl CoA reductase; AMPK: AMP-activated protein kinase; SREBP-1: sterol-regulatory element binding protein 1.

\section{References}

[1] Gaggini, M., Morelli, M., Buzzigoli, E., DeFronzo, R.A., Bugianesi, E. and Gastaldelli, A, "Non-Alcoholic Fatty Liver Disease (NAFLD) and Its Connection with Insulin Resistance, Dyslipidemia, Atherosclerosis and Coronary Heart Disease," Nutrients, 5(5). 1544-1560. 2013.

[2] Lee, J., Chung, D.S., Kang, J.H., and Yu, B.Y, "Comparison of Visceral Fat and Liver Fat as Risk Factors of Metabolic Syndrome," J Korean Med Sci, 27(2), 184-189. Feb. 2012.

[3] Bellentani, S., Scaglioni, F., Marino, M. and Bedogni, G, "Epidemiology of non-alcoholic fatty liver disease," Dig Dis, 28. 155-161. 2010.

[4] Lim, C.T., Kola, B. and Korbonits, M, "AMPK as a mediator of hormonal signalling," J Mol Endocrinol, 44. 87-97. 2010.
[5] Byeon, S.E., Lee, J., Kim, J.H., Yang, W.S., Kwak, Y.H., Kim, S.Y., Choung, E.S., Rhee, M.H., and Choi, J.Y, "Molecular mechanism of macrophage activation by red ginseng acidic polysaccharide from Korean red ginseng," Mediators of Inflammation, 2012(2012). 1-7. 2012,

[6] Choi, H., Kim T.H., Choi, T.Y., and Lee, M.S, "Ginseng for health care: a systematic review of randomized controlled trials in Korean literature," PLoS ONE, 8(4). e59978. Apr. 2013.

[7] Kim, J.H, "Cardiovascular Diseases and Panax ginseng: A Review on Molecular Mechanisms and Medical Applications," J Ginseng Res, 36(1). 16-26. Jan. 2012.

[8] Jung, J., Park, H.W., Hahn, Y., Hur, C.G., In, D., Chung, H.J., Liu, J. and Choi, D.W, "Discovery of genes for ginsenoside biosynthesis by analysis of ginseng expressed sequence tags," Plant cell reports, 22. 224-230. 2003.

[9] Yi, J.H., Kim, M.Y., Kim, Y.C., Jeong, W.S., Bae, D.W., Hur, J.M., Jun, M, "Change of ginsenoside composition in red ginseng processed with citric acid," Food Sci Biotechnol, 19. 647-653. 2010.

[10] Kim, S.O., Park, C.W., Moon, S., Lee, H.A., Kim, B., Lee, D.U., Lee, J.H. and Park, J, "Effects of high-hydrostatic pressure on ginsenoside concentrations in Korean red ginseng," Food Sci Biotechnol, 16. 848-853. 2007.

[11] Yuan, H.D., Kim, S.J., Quan, H.Y., Huang, B. and Chung, S.H, "Ginseng leaf extract prevents high fat diet-induced hyperglycemia and hyperlipidemia through AMPK activation," $J$ Ginseng Res, 34. 369-375. 2010.

[12] Ji, W. and Gong, B, "Hypolipidemic effects and mechanisms of Panax notoginseng on lipid profile in hyperlipidemic rats," $J$ Ethnopharmacol, 113. 318-324. 2007.

[13] Lee, H.S., Lee, H.J., Yu, H.J., Ju, D.W., Kim, Y., Kim, C.T., Kim, C.J., Cho, Y.J., Kim, N. and Choi, S.Y, "A comparison between high hydrostatic pressure extraction and heat extraction of ginsenosides from ginseng (Panax ginseng CA Meyer)," J Sci Food Agric, 91. 1466-1473. 2011.

[14] Lee, S., Lee, M.S., Kim, C.T., Kim, I.H. and Kim, Y, "Ginsenoside Rg3 reduces lipid accumulation with AMP-activated protein kinase (AMPK) activation in HepG2 Cells," Int J Mol Sci, 13. 5729-5739. 2012.

[15] Rozen, S., Skaletsky, H, Primer3 on the WWW for general users and for biologist programmers In Bioinformatics methods and protocols: methods in molecular biology. Edited by Misener, S., and Krawetz, S, Humana Press, New Jersey, 1999, 365-386.

[16] Livak, K.J. and Schmittgen, T.D, "Analysis of relative gene expression data using real-time quantitative PCR and the $2^{-\Delta \Delta C T}$ method," Methods, 25. 402-408. 2001.

[17] Ou, T.T., Hsu, M.J., Chan, K.C., Huang, C.N., Ho, H.H. and Wang, C.J, "Mulberry extract inhibits oleic acid-induced lipid accumulation via reduction of lipogenesis and promotion of hepatic lipid clearance," J Sci Food Agric, 91.2740-2748. 2011.

[18] Bhala, N., Jouness, R.I. and Bugianesi, E, "Epidemiology and natural history of patients with NAFLD," Current Pharmaceutical Design, 19(29). 5169-5176. 2013.

[19] Sohn, H., Lee, S. and Wee, J, "Flavor characteristics of Korean red ginseng," J Ginseng Res, 24. 148-152. 2000.

[20] Do Yeon Kim, J.S.P, Yuan, H.D. and Chung, S.H, "Fermented ginseng attenuates hepatic lipid accumulation and hyperglycemia through AMPK activation," Food Sci Biotechnol, 18. 172-178. 2009.

[21] Song, Y.B., An, Y.R., Kim, S.J., Park, H.W., Jung, J.W., Kyung, J.S., Hwang, S.Y. and Kim, Y.S, "Lipid metabolic effect of Korean red ginseng extract in mice fed on a high-fat diet," $J$ Sci Food Agric, 92. 388-396. 2012.

[22] Hillgartner, F.B., Salati, L.M. and Goodridge, A.G, "Physiological and molecular mechanisms involved in nutritional regulation of fatty acid synthesis," Physiol Rev, 75. 47-76. 1995.

[23] Goldstein, J.L. and Brown, M.S, "Regulation of the mevalonate pathway," Nature, 343. 425-30. 1990.

[24] Lee, Y.S., Cha, B.Y., Yamaguchi, K., Choi, S.S., Yonezawa, T., Teruya, T., Nagai, K. and Woo, J.T, "Effects of Korean white ginseng extracts on obesity in high-fat diet-induced obese mice," Cytotechnology, 62. 367-376. 2012.

[25] Muwalla, M.M, and Abuirmeileh, N.M, "Suppression of avian hepatic cholesterogenesis by dietary ginseng," The Journal of nutritional biochemistry, 1(10). 518-521. Oct. 1990.

[26] Hardie, D.G, "The AMP-activated protein kinase pathway-new players upstream and downstream," J Cell Sci, 117. 5479-5487. 2004. 
[27] Quan, H.Y., Yuan, H.D., Jung, M.S., Ko, S.K., Park, Y.G. and Chung, S.H, "Ginsenoside Re lowers blood glucose and lipid levels via activation of AMP-activated protein kinase in HepG2 cells and high-fat diet fed mice," Int J Mol Med, 29. 73. 2012.
[28] Kim, D.Y., Yuan, H.D., Chung, I.K. and Chung, S.H, "Compound $\mathrm{K}$, intestinal metabolite of ginsenoside, attenuates hepatic lipid accumulation via AMPK activation in human hepatoma cells," $J$ Agric Food Chem, 57. 1532-1537. 2009. 